\title{
Collaborative quality improvement reduced ventilator-associated pneumonia in a limited resource country
}

\author{
Akeau Unahalekhaka \\ Faculty of Nursing, Chiang Mai University, Thailand \\ doi:10.3396/ijic.V4s1.020.08
}

\begin{abstract}
Ventilator-associated pneumonia (VAP) is a hospital-acquired infection with the highest case fatality rate in Thailand. A collaborative approach among multidisciplinary professionals across 18 hospitals in three regions of Thailand had led to a considerable reduction in VAP among mechanically ventilated patients admitted in intensive care units. By applying the collaborative quality improvement concept, participating hospitals experienced a gradual decrease in the trend of the VAP rate per 1000 ventilator-days. Surveillance systems for VAP have improved and the approach had led to advances in the care of mechanically ventilated patients, resulting in the reduction of VAP incidence.
\end{abstract}

\section{Introduction}

Ventilator-associated pneumonia (VAP) is one of the most serious hospital-acquired infections among all secondary and tertiary care hospitals in Thailand with a high infection rate and case fatality rate. VAP prolongs the length of hospital stay and increases costs of treatment. There are many factors related to VAP problems, for example, inappropriate nurse to patient ratio; workload; nursing care practices; and limited space and resources.

Since VAP is a very complicated problem, prevention needs to be through a collaboration of multidisciplinary professionals not only within the hospital but also between hospitals. A team of 18 secondary and tertiary care hospitals in three regions of Thailand joined and worked together during February 2004 to May 2005 to effectively prevent VAP in intensive care units.

All together 253 hospital personnel participated in this project, including physicians, radiologists, anaesthesiologists, infection control nurses, intensive care unit(ICU) head nurses and nursing staff, pharmacists, microbiologists, nutritionists, physiotherapists and heads of central sterile supply department.

Method

\section{Collaborative quality improvement}

The entire project was divided into three phases:

Phase 1: Evaluation and improvement of the VAP surveillance system 
Phase 2: Application of the collaborative quality improvement concept to prevent VAP. All 18 hospital teams participated in the first workshop. The collaborative quality improvement concept was applied along with education on continuous quality improvement and prevention of VAP. During this workshop, surveillance systems were strengthened using the same definition, guideline and form.

After participating in the project workshop, each hospital team conducted its own activities. This included:

- Strengthening the VAP surveillance system;

- Reviewing the VAP prevention guidelines for the hospital and conducting gap analysis;

- Educating registered nurses on VAP diagnosis, VAP surveillance and prevention of VAP among mechanically ventilated patients;

- Strengthening hand hygiene practice.

Three months and nine months after the first workshop, regional workshops were conducted twice each for three regions. They allowed the teams to exchange experiences, report on the hospitals' progress and seek expert consultation.

Phase 3: Evaluation of the impact of applying the collaborative quality improvement concept in the prevention of VAP.

\section{Results}

The outcome indicator of the project was the reduction of ventilator-associated pneumonia in the ICU of participating hospitals. The VAP surveillance system was improved and the data was made more reliable

\section{VAP rates}

A comparison of VAP rates from active surveillance of each participating hospital from the beginning to the end of the project revealed a reduction of VAP in the majority of hospitals.

Among five hospitals in the central region, VAP rates decreased from 14.2 per 1000 ventilator-days to 4.7 (66.9\% reduction). Among five hospitals in the northern region, VAP rates decreased from 12.5 to 1.9, with $84.8 \%$ reduction, and among eight hospitals in the southern region, VAP rates reduced by $50.4 \%$ (from 13.3 to 6.6).

The trend of the VAP rate (per 1000 ventilator-days) of all 18 participating hospitals decreased gradually after applying the collaborative quality improvement concept. The linear decreasing trend of VAP incidence was statistically significant with a reduction of $6 \%$ (95\% Cl $1 \%, 6.8 \%$ ) per month. There is evidence of some success of the intervention during this observation time of 12 months.

Collaborative quality improvement led to advances in the caring of mechanically ventilated patients, resulting in the reduction of VAP incidence. Hospital personnel gained more understanding on continuous quality improvement, and got better in the way they worked as a team. They were willing to apply collaborative methods in solving other complicated hospital acquired infection problems.

\section{Communication and personnel}

There was rapid improvement for problems that were complicated and needed cooperation from personnel from multidisciplinary occupations. There was closer contact and better collaboration among personnel on both intra- and inter-department levels within the hospital.

Hospital personnel gained more knowledge on ventilator VAP and could apply the knowledge to their daily patient care. They also obtained the same clear and practicable best practice guidelines in prevention of VAP which all relevant hospital personnel could follow. This made them eagerly improve their quality of care continuously, making them feel much more confident in caring for mechanically-ventilated patients. Better care of hospital personnel also led to the reduction of VAP rates and the better quality of life of the patients.

Inter hospital collaboration was created among hospitals participating in the project. The collaborative concept was applied to other units of the hospitals. The hospital administrators were also interested in the project and supported their teams to work more conveniently. 


\section{Changes after implementation of the project}

The majority of participating hospital personnel made three main changes:

- Surveillance and reliable diagnosis of VAP with clear VAP definition: Clinicians should help diagnose VAP and implement an effective VAP surveillance system.

- Patient care practices: Personnel complied with VAP prevention guideline, standardised patient care practices and changes in many nursing procedures according to the guideline
- Personnel cooperation: Hospital personnel understood and realised the importance of VAP prevention. There were many relevant sub projects conducted in many units. Personnel clearly understood their roles and the hospital director supported them.

\section{Conclusions}

This project helped hospital teams work more effectively and rapidly. Care of mechanically ventilated patients improved, leading to reduction in the rate of VAP, which in turn meant saving patients lives, and reduction of costs of treatment and operation. 